\title{
The clinical significance of cutaneous metastasis and paraneoplastic skin lesions in ovarian cancer. A rare but not minor manifestation
}

\author{
Machairiotis Nikolaos, Thomakos Nikolaos, Malakasis Anastasios, Rodolakis Alexandros \\ First Department of Obstetrics and Gynecology, Alexandra Hospital, Gynecologic Oncology Unit, \\ University of Athens, Athens, Greece

\begin{abstract}
Correspondence
Nikolaos Machairiotis First Department of Obstetrics and Gynecology - Gynecologic Oncology Unit Alexandra General Hospital, University of Athens, Greece, Tel: 00306945893801, E-mail: nikolaosmachairiotis@gmail.com
\end{abstract}

\begin{abstract}
Ovarian cancer results in the formation and proliferation of abnormal cells that have the ability to proliferate faster than normal/typical ovarian cells and then spread to other parts of the body either in a local or in a systemic way. The areas to which the metastases of ovarian cancer can be located include the lining of the abdomen, lining of the bowel and bladder, lymph nodes, lungs, liver and in very rare cases the skin. We performed a review of the literature in order to find and document the cutaneous manifestations of the different types of ovarian cancer. Skin is a rather unusual organ for the localization of metastatic ovarian cancer but it must not be overseen. Cutaneous metastasis from carcinoma are relatively uncommon in clinical practice but they are very important to recognize. Cutaneous metastasis may herald the diagnosis of internal malignancy, and early recognition can lead to accurate and prompt diagnosis and timely treatment. However, a high index of suspicion is required because the clinical findings may be subtle. Our understanding of skin disease remains mainly descriptive rather than pathophysiologic. The variation and the multipotent skin expression of the ovarian as well as other intrabdominal, supradiaphragmatic malignancies reveal their similarity to autoimmune diseases, leading to the inert question if it is immunologic modulated.
\end{abstract}

Key words: skin lesions, ovarian cancer, metastasis, paraneoplasia

\section{Introduction}

Ovarian cancer is neoplastic process leading to the formation and proliferation of abnormal cells that have the ability to procreate faster than normal/typical ovarian cells and then spread to other parts of the body either in a local or in a systemic way ${ }^{1}$. When this oncogenic process is in the primary stages, the symptomatology is either absent or vague and becomes more noticeable as the disease progresses ${ }^{2}$. 
These first general symptoms usually include among others bloating, pelvic pain, abdominal swelling, and loss of appetite ${ }^{3,2}$. Ovarian cancer has a clear predilection for metastasis to the omentum and peritoneal surfaces, but the underlying mechanisms involved in ovarian cancer spread are not well understood. Despite this fact many studies have shown that the hematogenous mechanism of metastasis is the dominant way of ovarian cancer spreading 4 .

The areas to which the metastases of ovarian cancer can be located include the lining of the abdomen, lining of the bowel and bladder, lymph nodes, lungs, liver and in very rare cases the skin ${ }^{3,5}$.

It is also important to refer to another very rare manifestation of ovarian cancer and that is the skin paraneoplastic disease that can accompany this tumor. As it is widely known paraneoplastic syndromes are clinical entities deriving from malignancies and are expressed at a distant site from the primary tumor or its secondary manifestations. Since skin is a rather unusual organ for the localization of metastatic ovarian cancer, we performed a review of the literature in order to find and document the cutaneous manifestations.

\section{Materials and Methods}

We performed a research in PubMed using the key words "cutaneous manifestations of ovarian cancer", "ovarian cancer metastasis", " skin metastasis and ovarian cancer", "paraneoplastic skin lesion in ovarian cancer".

\section{Results}

Flam F et al. presented case of a 78 year old woman with a medical history of breast cancer that was treated with tamoxifen. The patient presented subcutaneous metastasis which was initially considered to be a metastatic locus deriving from the primary breast cancer. The further follow up of the tumor, revealed its ovarian origin. This case report suggests the use of hormonal agents for the treatment of these distant metastatic sites ${ }^{6}$.

Local skin metastasis from trocar implantation is another issue that surgeons had to deal with. In the study by van Dam P. et. al. it was suggested that chemotherapy administration and local excision of the trocar trajectories can reduce local skin metastasis from advanced ovarian cancer after laparoscopic surgery ${ }^{7}$.

Traiman P et al. presented the case of a 37 year old woman presented with papillary cystadenocarcinoma of the ovary. The patient did not wish to receive treatment upon diagnosis. She was readmitted for the further treatment after six years. At the admission time, the follow-up revealed an extremely large, cutaneous, cauliflower-type of metastasis located in the lower abdominal wall and measuring $20 \times 20 \mathrm{~cm}$. A neoadjuvant therapeutic schema was administered (chemotherapy and local radiotherapy) before the laparotomy ${ }^{8}$.

In the case series ( 9 ovarian cancer cases with skin metastases) reported by Cormio G. et. al. was observed that skin involvement occurs rarely in ovarian cancer patients and usually at an advanced stage of the disease. Moreover; the prognosis after the cutaneous manifestations of the disease is usually poor. In these cases early diagnosis and documentation of the skin metastases as the evolution of ovarian neoplastic disease is the most important prognostic factor associated with survival ${ }^{9}$.

In the study by Touraud J. P. et. al. umbilical metastasis as a manifestation of ovarian cancer was reported. It has been observed that gynaecological cancers are the second most frequent cause of umbilical metastasis, with ovarian cancers being the most common (34\% of cases). Again, survival is reported with a poor prognosis (mean survival: 1012 months). However, patient survival time could be increased with aggressive therapy, i.e., surgery 
combined with chemotherapy, radiotherapy and immunotherapy ${ }^{10}$.

In the study conducted by Tsai H-W. et.al. two cases of Sister Mary Joseph's nodules (umbilical metastases) were presented. It was suggested that in addition to tumor marker CA125, a physical examination assisted by modern imaging evaluation, should be considered the best method for detecting early recurrence in patients with previously treated ovarian cancer ${ }^{11}$.

Moreover; even if an umbilical metastasis usually indicates advanced disease with disseminating peritoneal spread, this finding on its own does not imply inoperable or incurable disease. In the case by Lalich D. et. al. for the first time a cutaneous metastasis of a visceral neoplasm mimicking a primary pilomatrical neoplasm was presented. The authors described a metastasis of an ovarian carcinoma with shadow cells to the skin that was initially misinterpreted as a pilomatricoma ${ }^{12}$.

Nakamura S. et. al. reported a case of cutaneous lymphangitis carcinomatosis secondary to underlying extra-ovarian primary peritoneal papillary serous carcinoma ${ }^{13}$.

Yilmaz et al described a single case of a 69 year old woman who presented a serious papillary adenocarcinoma of the ovary with pleural metastasis which was treated with adjuvant therapy (chemotherapy and surgery) and the post-treatment follow-up for the first year was disease free. In the period of time followed, she presented an oedema of her right thigh, a cellulitis like syndrome and erythematous nodules located at the lower abdominal wall and the upper leg skin. This entity was correlated with poor prognosis ${ }^{14}$.

\section{Discussion}

Cutaneous metastases from carcinoma are relatively uncommon in clinical practice, but they are very important to recognize. Cutaneous metastasis may herald the diagnosis of internal malignancy, and early recognition can lead to accurate and prompt diagnosis and timely treatment. However, a high index of suspicion is required because the clinical findings may be subtle.

Our understanding of neoplastic manifestations of skin disease remains mainly descriptive rather than pathophysiologic.

Although ovarian cancer secretes or responds to many cytokines and chemokines such as transforming growth factor-b, insulin-like growth factor, some sub- types of fibroblast growth factor, epidermal growth factor and / or vascular endothelial growth factor, the statistical significance of the clinical and preclinical studies conducted on this topic showed; that the molecular intracellular or signaling pathway of this entity and its cutaneous manifestations remains unclear and undefined, leading in an uncertainty to the treatment ${ }^{15}$.

The pathophysiological mechanism mentioned above can also cause the expression of paraneoplastic syndromes. The cutaneous paraneoplastic syndromes are a group of skin lesions that may appear in every stage of the neoplastic disease, which means that they can be present even before the presence of the primary tumor and therefore the high clinical suspicion can lead to early discovery and treatment of the underlying malignancy.

The term paraneoplastic syndromes refers to variety of signs and symptoms located at distant sites from the primary tumor and which are not directly caused by the tumor invasion nor its metastases nor the destruction of normal tissue. These syndromes may be the result of hormonal and cytokine secretion of the tumor or they can be a malignancyinduced immune response. However, in many cases, the cause and physiopathology of paraneoplastic syndromes is unknown ${ }^{16}$.

Various paraneoplastic syndromes have been described in the literature so far, including endocrine, 
gastrointestinal, neurologic, dermatologic, renal, hematologic and rheumatologic manifestations of the disease. Since the paraneoplastic syndrome can be the very first sign of the tumor or its recurrence, prompt recognition and evaluation is crucial for early cancer detection.

The diagnosis of a paraneoplastic syndrome, is an exclusion diagnosis; in other words tumor invasion, obstruction, vascular compromise, cancertreatment toxicity and fluid or electrolyte disorders need to be excluded as causes of the clinical manifestation that is named after the term paraneoplastic syndrome ${ }^{16,17}$.

Despite their rarity it is considered important to refer to the skin lesions reported in the literature as paraneoplastic manifestations of gynecologic malignancies. These include acanthosis nigricans, in association with cancers of the ovary and cervix (Arnold et al. 2001; Sabir et al. 1999; Rigel and Jacobs 1980). Leser-Tre'lat, sign associated with cancers of the cervix and ovary (Ashour et al. 1997), Erythema gyratum repens, in association with uterine cancer (Kurzrock and Cohen 1995; Boyd et al. 1992), Hypertrichosis lanuginosa acquisita, referred also as 'malignant down' and most commonly associated with neoplasms of the uterus (McKenna et al. 1992; Hovenden 1993) and extensive pruritic eruption described by Rodolakis et al and was associated with ovarian mucinous cystadenocarcinoma ${ }^{16}$.

The pathogenesis of this neoplastic symptomatology is not defined yet. The systematic manifestation of the disease indicates that the immunomodulation caused by the cancer itself; is the reason of the cutaneous manifestations ${ }^{18}$.

The treatment of these extra-abdominal metastases of ovarian cancers is not standardized. The application of chemotherapy, which is mainly palliative in the cases of these patients, is the necessity of considering the therapeutic option of sympto- matic management of cutaneous metastases. According to that, treatment options depend on the patients symptoms and the level of comfort as well as the number and localization of the lesions. In cases of local cutaneous disease, surgical resection may be a therapeutic option ${ }^{16}$. The decision making strategy and algorithm in the cases of these patients is complicated, because the treatment of recurrent ovarian cancer is palliative; in this aspect performing a surgical excision can be a therapeutic option in some cases for patients meeting some criteria as the good general condition and the life expectancy. Skin lesions located on the extremities may be safer to be operatively removed than abdominal cutaneous lesions, which are in their majority associated with intra-abdominal lesions. Another therapeutic option for extensive cutaneous metastasis is considered to be the electrocoagulation or external of the lesions aiming in local control of the disease and alleviation of the pain, minimizing of hemorrhage and infection ${ }^{19-21}$.

\section{Conclusion}

As mentioned above, cutaneous manifestations of ovarian neoplastic disease, either in the form of paraneoplastic syndrome or metastasis, are extremely rare. The variation and the multipotent skin expression of the ovarian as well as other intraabdominal, supradiaphragmatic malignancies reveal their similarity to autoimmune diseases, leading to the inert question if it is immunologic modulated.

In conclusion, the scientific community should concentrate on the pathogenesis of the cutaneous manifestations of ovarian epithelial malignancies.

\section{Conflict of Interest}

None to declare 


\section{Acknowledgments}

All authors contributed equally to this wok

\section{References}

1. Roett MA, Evans P. Ovarian cancer: an overview. Am Fam Physician. 2009;80(6):609-16.

2. Ebell MH, Culp MB, Radke TJ. A Systematic Review of Symptoms for the Diagnosis of Ovarian Cancer. Am J Prev Med. 2016;50(3):384-94.

3. Martin VR. Ovarian cancer: an overview of treatment options. Clin J Oncol Nurs. 2007;11(2):201-7.

4. Pradeep S, Kim SW, Wu SY, Nishimura M, Chaluvally-Raghavan P, Miyake T, et al. Hematogenous metastasis of ovarian cancer: rethinking mode of spread. Cancer Cell. 2014;26(1):77-91.

5. Ruddon RW. Cancer biology (4th ed.). Oxford: Oxford University Press; 2007.

6. Flam F, Skoog L, Wilking N. Subcutaneous metastasis from an ovarian carcinoma disappeared following tamoxifen therapy. Eur J Obstet Gynecol Reprod Biol. 1996;64(2):225-6.

7. van Dam PA, DeCloedt J, Tjalma WA, Buytaert P, Becquart D, Vergote IB. Trocar implantation metastasis after laparoscopy in patients with advanced ovarian cancer: can the risk be reduced? Am J Obstet Gynecol. 1999;181(3):53641.

8. Traiman P, de Luca LA, Bacchi CE. An extremely large, cauliflower-type, cutaneous metastasis of ovarian cancer associated with good prognosis. Gynecol Oncol. 1994;53(2):239-41.

9. Cormio G, Capotorto M, Di Vagno G, Cazzolla A, Carriero C, Selvaggi L. Skin metastases in ovarian carcinoma: a report of nine cases and a review of the literature. Gynecol Oncol. 2003;90(3):682-5.

10. Touraud JP, Lentz N, Dutronc Y, Mercier E, Sagot P, Lambert D. [Umbilical cutaneous metastasis (or Sister Mary Joseph's nodule) disclosing an ovarian adenocarcinoma]. Gynecol Obstet Fertil. 2000;28(10):719-21.

11. Tsai HW, Yuan CC, Wang PH. Umbilicus as the only site of metastasis in recurrent ovarian cancer. J Chin Med Assoc. 2006;69(5):233-5.

12. Lalich D, Tawfik O, Chapman J, Fraga G. Cutaneous metastasis of ovarian carcinoma with shadow cells mimicking a primary pilomatrical neoplasm. Am J Dermatopathol. 2010;32 (5):500-4.

13. Nakamura S, Hashimoto Y, Nishi K, Mizumoto T, Takahashi H, Iizuka H. Cutaneous lymphangitis carcinomatosis metastasis of extra-ovarian primary peritoneal carcinoma. Acta Derm Venereol. 2012;92(6):639-40.

14. Yilmaz Z, Bese T, Demirkiran F, Ilvan S, Sanioglu C, Arvas M, et al. Skin metastasis in ovarian carcinoma. Int J Gynecol Cancer. 2006;16 Suppl 1:414-8.

15. Scheinfeld N. A review of the cutaneous paraneoplastic associations and metastatic presentations of ovarian carcinoma. Clin Exp Dermatol. 2008;33(1):10-5.

16. Rodolakis A, Thomakos N, Protopappas A, Doublis D, Sotiropoulou M, Milingos S. Ovarian mucinous cystadenocarcinoma of low malignant potential presented as a paraneoplastic skin lesion. J Obstet Gynaecol. 2005;25(6):624-6.

17. Ashour AA, Verschraegen CF, Kudelka AP, Kavanagh JJ. Paraneoplastic syndromes of gynecologic neoplasms. J Clin Oncol. 1997;15(3) :1272-82.

18. Kovacs KA, Kenessey I, Timar J. Skin metastasis of internal cancers: a single institution experience. Pathol Oncol Res. 2013;19(3):515-20.

19. Patsner B, Mann WJ, Chumas J, Loesch M. Herpetiform cutaneous metastases following negative second look laparatomy for ovarian adenocarcinoma. Arch Gynecol Obstet. 1988;244(1):63-7. 
20. Tapley ND. Radiation therapy with the electron beam. Semin Oncol. 1981;8(1):49-58.

21. Kim MK, Kim SH, Lee YY, Choi CH, Kim TJ, Lee JW, et al. Metastatic skin lesions on lower extremities in a patient with recurrent serous papillary ovarian carcinoma: a case report and literature review. Cancer Res Treat. Received 1-12-2017 2012;44(2):142-5. 